ANNALES

POLONICI MATHEMATICI

$82.1(2003)$

\title{
Bifurcation theorems for nonlinear problems with lack of compactness
}

\author{
by Francesca Faraci (Catania) and Roberto Livrea (Messina)
}

Abstract. We deal with a bifurcation result for the Dirichlet problem

$$
\left\{\begin{array}{l}
-\Delta_{p} u=\frac{\mu}{|x|^{p}}|u|^{p-2} u+\lambda f(x, u) \quad \text { a.e. in } \Omega, \\
u_{\mid \partial \Omega}=0 .
\end{array}\right.
$$

Starting from a weak lower semicontinuity result by E. Montefusco, which allows us to apply a general variational principle by B. Ricceri, we prove that, for $\mu$ close to zero, there exists a positive number $\lambda_{\mu}^{*}$ such that for every $\left.\lambda \in\right] 0, \lambda_{\mu}^{*}[$ the above problem admits a nonzero weak solution $u_{\lambda}$ in $W_{0}^{1, p}(\Omega)$ satisfying $\lim _{\lambda \rightarrow 0^{+}}\left\|u_{\lambda}\right\|=0$.

1. Introduction. In the present paper we are interested in the existence of solutions for the Dirichlet problem

$\left(P_{\lambda, \mu}\right) \quad\left\{\begin{array}{l}-\Delta_{p} u=\frac{\mu}{|x|^{p}}|u|^{p-2} u+\lambda f(x, u) \quad \text { a.e. in } \Omega, \\ u_{\mid \partial \Omega}=0,\end{array}\right.$

where $\Omega$ is a bounded open subset of $\mathbb{R}^{n}(n \geq 2)$ containing the origin, $1<p<N, f: \Omega \times \mathbb{R} \rightarrow \mathbb{R}$ is a Carathéodory function with $f(x, 0)=0$, and $\lambda, \mu$ are two parameters respectively positive and nonnegative.

The presence of the term $\mu /|x|^{p}$ does not allow us to apply the classical variational approach. The Hardy inequality ensures that $W_{0}^{1, p}(\Omega)$ is continuously, but not compactly embedded in $L^{p}(\Omega)$ with respect to the weight $|x|^{-p}$. Because of this lack of compactness we are not able to obtain the weak lower semicontinuity of the energy functional via the classical De Giorgi theorem.

The problem

$$
\left\{\begin{array}{l}
-\Delta_{p} u=\frac{\mu}{|x|^{p}}|u|^{p-2} u+f(x) \quad \text { a.e. in } \Omega, \\
u_{\mid \partial \Omega}=0
\end{array}\right.
$$

2000 Mathematics Subject Classification: 35B32, 35J60.

Key words and phrases: bifurcation point, $p$-Laplacian, critical points. 
is studied in [3], where $f \in W^{-1, p^{\prime}}(\Omega)$ and $\mu<H, H$ being the best constant in the Hardy inequality. In particular, starting from the coercivity and homogeneity of the energy functional, the authors of [3] are able to prove the required compactness property by finding a minimizing sequence converging to a global minimum.

The authors of [1] are interested in minima of the nondifferentiable functional

$$
J(u)=\int_{\Omega} j(x, \nabla u) d x-a \int_{\Omega} \frac{|u|^{2}}{|x|^{2}} d x-\int_{\Omega} f(x) u(x) d x,
$$

where $f \in L^{2}(\Omega)$ and $j(x, \xi)$ is a convex function with respect to $\xi$, satisfying

$$
\alpha|\xi|^{2} \leq j(x, \xi) \leq \beta|\xi|^{2}
$$

for every $\xi \in \mathbb{R}^{n}$, a.e. in $\Omega$. With no information about the weak lower semicontinuity of the functional, they state the existence of a global minimum using a truncation approach.

We refer moreover to the recent papers [2] and [4] for a complete survey of the topic. The authors of [4] deal with the problem

$$
\left\{\begin{array}{l}
-\Delta_{p} u=\frac{\mu}{|x|^{s}}|u|^{q-2} u+\lambda|u|^{r-2} u \quad \text { a.e. in } \Omega, \\
u_{\mid \partial \Omega}=0
\end{array}\right.
$$

where $0 \leq s \leq p<n, q \leq p^{\star}(s)=(p-s) /(n-p) p$. In particular in the case $s=q=p, p<r<p^{\star}$ they obtain infinitely many solutions, at least one of them being positive, for any $\lambda>0$ and $0<\mu<H$. We notice that the main assumption in that paper is a bound on $r$, which is incompatible with our hypothesis on the nonlinearity at zero (see condition (3)).

Here we propose a novel approach to the subject. In particular, combining a weak lower semicontinuity result by E. Montefusco [5] with a recent variational principle by B. Ricceri [6] we establish a bifurcation theorem for problem $\left(P_{\lambda, \mu}\right)$ just assuming a suitable behaviour of $f(x, t)$ at zero. Moreover, we prove that if $\lambda$ is sufficiently small, then the energy functional related to the problem is negative and decreasing on the solutions.

Finally, using the same technique, we obtain an analogous result for the problem

$$
\left(P_{\lambda, \mu}^{*}\right) \quad\left\{\begin{array}{l}
-\Delta_{p} u=\mu\left(\int_{\Omega}|u|^{p^{*}}\right)^{p / p^{*}-1}|u|^{p^{*}-2} u+\lambda f(x, u) \quad \text { a.e. in } \Omega, \\
u_{\mid \partial \Omega}=0,
\end{array}\right.
$$

where $n, p$ and $f$ are as in problem $\left(P_{\lambda, \mu}\right), \Omega$ is a bounded open subset of $\mathbb{R}^{n}$ and $p^{*}=n p /(n-p)$. 
2. Preliminaries. Assume the following growth condition on $f$ : there exist two positive constants $a, q$ with

$$
q<\frac{n(p-1)+p}{n-p}
$$

and a nonnegative constant $b$ such that

$$
|f(x, \xi)| \leq a|\xi|^{q}+b
$$

for every $\xi \in \mathbb{R}$ and a.e. in $\Omega$.

Denote by $X$ the space $W_{0}^{1, p}(\Omega)$ endowed with the norm

$$
\|u\|=\left(\int_{\Omega}|\nabla u(x)|^{p} d x\right)^{1 / p} .
$$

Let us recall the Hardy inequality

$$
\int_{\Omega} \frac{|u(x)|^{p}}{|x|^{p}} d x \leq \frac{1}{H} \int_{\Omega}|\nabla u(x)|^{p} d x,
$$

where $\Omega$ is an open set in $\mathbb{R}^{n}$ containing the origin and $H$ is the best constant in the inclusion of $W_{0}^{1, p}(\Omega)$ in $L^{p}(\Omega)$ with weight $|x|^{-p}$. In particular, when $\Omega$ is a ball, $H=((n-p) / p)^{p}$ (see [3]).

For each $u \in X$ and $\mu \in \mathbb{R}$ put

$$
\begin{aligned}
\mathcal{H}_{\mu}(u) & =\frac{1}{p} \int_{\Omega}|\nabla u(x)|^{p} d x-\frac{\mu}{p} \int_{\Omega} \frac{|u(x)|^{p}}{|x|^{p}} d x, \\
\Phi(u) & =-\int_{\Omega}\left(\int_{0}^{u(x)} f(x, \xi) d \xi\right) d x .
\end{aligned}
$$

In [5] it is shown that $\mathcal{H}_{\mu}$ is a well defined and continuously Gateaux differentiable functional in $X$. Moreover, if $\mu \in\left[0, H\left[\right.\right.$, then $\mathcal{H}_{\mu}$ is weakly lower semicontinuous and coercive.

Standard arguments show that $\Phi$ is a well defined and continuously Gateaux differentiable functional whose Gateaux derivative is a compact operator from $X$ to $X^{*}$.

A weak solution of problem $\left(P_{\lambda, \mu}\right)$ is any $u \in X$ such that

$$
\begin{aligned}
& \int_{\Omega}|\nabla u(x)|^{p-2} \nabla u(x) \cdot \nabla v(x) d x-\mu \int_{\Omega} \frac{|u(x)|^{p-2}}{|x|^{p}} u(x) v(x) d x \\
& -\lambda \int_{\Omega} f(x, u(x)) v(x) d x=0 \quad \text { for all } v \in X .
\end{aligned}
$$

For each $\lambda, \mu \in \mathbb{R}$, consider the functional $J_{\lambda, \mu}: X \rightarrow \mathbb{R}$ defined by

$$
J_{\lambda, \mu}(u)=\mathcal{H}_{\mu}(u)+\lambda \Phi(u)
$$

and observe that it is the energy functional related to $\left(P_{\lambda, \mu}\right)$. 
Finally, for the reader's convenience, we recall the main tool that we will use. It is due to B. Ricceri and it can be stated as follows:

Theorem A ([6, Theorem 2.5]). Let $X$ be a reflexive real Banach space, and let $\Phi, \Psi: X \rightarrow \mathbb{R}$ be two sequentially weakly lower semicontinuous functionals. Assume also that $\Psi$ is (strongly) continuous and $\lim _{\|x\| \rightarrow+\infty} \Psi(x)$ $=+\infty$. For each $\varrho>\inf _{X} \Psi$, put

$$
\varphi(\varrho)=\inf _{x \in \Psi^{-1}(]-\infty, \varrho[)} \frac{\Phi(x)-\inf _{\mathrm{cl}_{w} \Psi^{-1}(]-\infty, \varrho[)} \Phi}{\varrho-\Psi(x)},
$$

where $\mathrm{cl}_{w}$ is the closure in the weak topology. Then, for each $\varrho>\inf _{X} \Psi$ and each $\lambda>\varphi(\varrho)$, the restriction of the functional $\Phi+\lambda \Psi$ to $\Psi^{-1}(]-\infty, \varrho[)$ has a global minimum.

\section{Main result}

Theorem 3.1. Let $f: \Omega \times \mathbb{R} \rightarrow \mathbb{R}$ be a Carathéodory function with $f(x, 0)=0$, satisfying condition (1). Assume that there are a nonempty open set $D \subseteq \Omega$ and a set $B \subseteq D$ of positive measure such that

$$
\begin{gathered}
\limsup _{\xi \rightarrow 0^{+}} \frac{\inf _{x \in B} \int_{0}^{\xi} f(x, t) d t}{|\xi|^{p}}=+\infty, \\
\liminf _{\xi \rightarrow 0^{+}} \frac{\inf _{x \in D} \int_{0}^{\xi} f(x, t) d t}{|\xi|^{p}}>-\infty .
\end{gathered}
$$

Then for every $\mu \in\left[0, H\left[\right.\right.$ there exists a positive number $\lambda_{\mu}^{*}$ such that for every $\lambda \in] 0, \lambda_{\mu}^{*}\left[\right.$ problem $\left(P_{\lambda, \mu}\right)$ admits a nonzero weak solution $u_{\lambda}$ in $W_{0}^{1, p}(\Omega)$. Moreover,

$$
\lim _{\lambda \rightarrow 0^{+}}\left\|u_{\lambda}\right\|=0
$$

and the function $\lambda \mapsto J_{\lambda, \mu}\left(u_{\lambda}\right)$ is negative and decreasing in $] 0, \lambda_{\mu}^{*}[$.

Proof. Fix $\mu \in\left[0, H\left[\right.\right.$. We want to apply Theorem A, where $X=W_{0}^{1, p}(\Omega)$, $\Psi=\mathcal{H}_{\mu}$ and $\Phi$ is the functional introduced in Section 2 .

Since $\mu \in[0, H[$, we have already observed in Section 2 that $\Phi$ and $\Psi$ are two sequentially weakly lower semicontinuous and continuously Gateaux differentiable functionals. Moreover, $\Psi$ is coercive and clearly $\inf _{u \in X} \Psi(u)$ $=0$.

Let $\bar{\varrho}>0$ be such that $\varphi(\bar{\varrho})>0$ and put $\lambda_{\mu}^{*}=1 / \varphi(\bar{\varrho})$. Thanks to Theorem A, for every $\lambda \in] 0, \lambda_{\mu}^{*}\left[\right.$ there exists $u_{\lambda} \in \Psi^{-1}(]-\infty, \bar{\varrho}[)$ such that

$$
\Phi^{\prime}\left(u_{\lambda}\right)+\frac{1}{\lambda} \Psi^{\prime}\left(u_{\lambda}\right)=0
$$

and, in particular, $u_{\lambda}$ is a global minimum of the restriction of $\Phi+\frac{1}{\lambda} \Psi$ to $\Psi^{-1}(]-\infty, \bar{\varrho}[)$. 
Fix $\lambda \in] 0, \lambda_{\mu}^{*}\left[\right.$; we will prove that $u_{\lambda} \neq 0$. To this end, let us prove that

$$
\liminf _{\|u\| \rightarrow 0^{+}} \frac{\Phi(u)}{\Psi(u)}=-\infty \text {. }
$$

Thanks to (3) we can fix a sequence $\left\{\xi_{k}\right\}$ in $\mathbb{R}^{+}$converging to zero and two constants $\delta$ and $\Gamma$ with $\delta>0$ such that

$$
\lim _{k \rightarrow+\infty} \frac{\inf _{x \in B} \int_{0}^{\xi_{k}} f(x, t) d t}{\left|\xi_{k}\right|^{p}}=+\infty
$$

and

$$
\inf _{x \in D} \int_{0}^{\xi} f(x, t) d t \geq \Gamma|\xi|^{p}
$$

for all $\xi \in[0, \delta]$. Next, fix a set $C \subset B$ of positive measure and a function $v \in X$ such that $v(x) \in[0,1]$ for all $x \in \Omega, v(x)=1$ for all $x \in C$, and $v(x)=0$ for all $x \in \Omega \backslash D$. Let $Q>0$, put

$$
\|\| v \|\left.\right|^{p}=\int_{\Omega} \frac{|v(x)|^{p}}{|x|^{p}} d x
$$

and consider a positive number $T$ with

$$
Q<\frac{T \operatorname{meas}(C)+\Gamma \int_{D \backslash C}|v(x)|^{p} d x}{\frac{1}{p}\|v\|^{p}-\frac{\mu}{p}\|v\|^{p}} .
$$

Then there is $\nu \in \mathbb{N}$ such that $\xi_{k}<\delta$ and

$$
\inf _{x \in B} \int_{0}^{\xi_{k}} f(x, t) d t \geq T\left|\xi_{k}\right|^{p}
$$

for all $k>\nu$. Now, for each $k>\nu$, one has

$$
\begin{aligned}
-\frac{\Phi\left(\xi_{k} v\right)}{\Psi\left(\xi_{k} v\right)} & =\frac{\int_{C}\left(\int_{0}^{\xi_{k}} f(x, t) d t\right) d x+\int_{D \backslash C}\left(\int_{0}^{\xi_{k} v(x)} f(x, t) d t\right) d x}{\frac{1}{p}\left\|\xi_{k} v\right\|^{p}-\frac{\mu}{p}\left\|\xi_{k} v \mid\right\|^{p}} \\
& \geq \frac{T \operatorname{meas}(C)+\Gamma \int_{D \backslash C}|v(x)|^{p} d x}{\frac{1}{p}\|v\|^{p}-\left.\frac{\mu}{p}\|v\|\right|^{p}}>Q .
\end{aligned}
$$

From (6), clearly (5) follows. Hence, there is a sequence $\left\{w_{k}\right\}$ in $X$ converging to zero such that for $k$ large enough we have $w_{k} \in \Psi^{-1}(]-\infty, \bar{\varrho}[)$, and

$$
\Phi\left(w_{k}\right)+\frac{1}{\lambda} \Psi\left(w_{k}\right)<0 .
$$

Since $u_{\lambda}$ is a global minimum of the restriction of $\Phi+\frac{1}{\lambda} \Psi$ to $\Psi^{-1}(]-\infty, \bar{\varrho}[)$, we can conclude that 


$$
\Phi\left(u_{\lambda}\right)+\frac{1}{\lambda} \Psi\left(u_{\lambda}\right)<0=\Phi(0)+\frac{1}{\lambda} \Psi(0)
$$

so that $u_{\lambda} \neq 0$.

Observing that the weak solutions of problem $\left(P_{\lambda, \mu}\right)$ are exactly the critical points of the functional $J_{\lambda, \mu}$ and that

$$
J_{\lambda, \mu}=\lambda\left(\Phi+\frac{1}{\lambda} \Psi\right)
$$

one finds that the first part of our theorem is completely proved.

Since $\Psi$ is coercive and $u_{\lambda} \in \Psi^{-1}(]-\infty, \bar{\varrho}[)$ for every $\left.\lambda \in\right] 0, \lambda_{\mu}^{*}[$, there exists a positive number $L$ such that

$$
\left\|u_{\lambda}\right\| \leq L
$$

for every $\lambda \in] 0, \lambda_{\mu}^{*}\left[\right.$. Therefore, since $\Phi^{\prime}$ is a compact operator, there exists a positive number $M$ such that

$$
\left|\int_{\Omega} f\left(x, u_{\lambda}(x)\right) u_{\lambda}(x) d x\right| \leq\left\|\Phi^{\prime}\left(u_{\lambda}\right)\right\|_{X^{*}}\left\|u_{\lambda}\right\| \leq M \cdot L^{2}
$$

for every $\lambda \in] 0, \lambda_{\mu}^{*}[$.

By $(4), J_{\lambda}^{\prime}\left(u_{\lambda}\right)=0$ for every $\left.\lambda \in\right] 0, \lambda_{\mu}^{*}\left[\right.$ and in particular $\left(J_{\lambda}^{\prime}\left(u_{\lambda}\right)\right)\left(u_{\lambda}\right)=0$, that is,

$$
p \cdot \Psi\left(u_{\lambda}\right)=\left\|u_{\lambda}\right\|^{p}-\mu\|\| u_{\lambda} \|\left.\right|^{p}=\lambda \int_{\Omega} f\left(x, u_{\lambda}(x)\right) u_{\lambda}(x) d x
$$

for every $\lambda \in] 0, \lambda_{\mu}^{*}[$. Hence

$$
\lim _{\lambda \rightarrow 0^{+}} \Psi\left(u_{\lambda}\right)=0 .
$$

Finally, putting together (2) and (9) yields

$$
\frac{1}{p}\left\|u_{\lambda}\right\|^{p} \leq \Psi\left(u_{\lambda}\right)+\frac{\mu}{p \cdot H}\left\|u_{\lambda}\right\|^{p}
$$

for every $\lambda \in] 0, \lambda_{\mu}^{*}[$, hence

$$
\left\|u_{\lambda}\right\|^{p} \leq \frac{p \cdot H}{H-\mu} \Psi\left(u_{\lambda}\right)
$$

for every $\lambda \in] 0, \lambda_{\mu}^{*}[$ and

$$
\lim _{\lambda \rightarrow 0^{+}}\left\|u_{\lambda}\right\|=0 .
$$

From (7) and (8) it follows that the function $\lambda \mapsto J_{\lambda, \mu}\left(u_{\lambda}\right)$ is negative in ] $0, \lambda_{\mu}^{*}\left[\right.$. Finally, if we fix $\left.\lambda_{1}, \lambda_{2} \in\right] 0, \lambda_{\mu}^{*}\left[\right.$ with $\lambda_{1}<\lambda_{2}$ and put

$$
\begin{aligned}
& m_{\lambda_{1}}=\Phi\left(u_{\lambda_{1}}\right)+\frac{1}{\lambda_{1}} \Psi\left(u_{\lambda_{1}}\right)=\inf _{v \in \Psi^{-1}(]-\infty, \bar{\varrho}[)}\left(\Phi(v)+\frac{1}{\lambda_{1}} \Psi(v)\right), \\
& m_{\lambda_{2}}=\Phi\left(u_{\lambda_{2}}\right)+\frac{1}{\lambda_{2}} \Psi\left(u_{\lambda_{2}}\right)=\inf _{v \in \Psi^{-1}(]-\infty, \bar{\varrho}[)}\left(\Phi(v)+\frac{1}{\lambda_{2}} \Psi(v)\right),
\end{aligned}
$$


then

$$
J_{\lambda_{1}, \mu}\left(u_{\lambda}\right)=\lambda_{1} m_{\lambda_{1}}>\lambda_{2} m_{\lambda_{1}} \geq \lambda_{2} m_{\lambda_{2}}=J_{\lambda_{2}, \mu}\left(u_{\lambda_{2}}\right) .
$$

Hence, $\lambda \mapsto J_{\lambda, \mu}\left(u_{\lambda}\right)$ is decreasing in $] 0, \lambda_{\mu}^{*}[$ and the proof is complete.

REMARK 1. Observe that Theorem 3.1 is a bifurcation result. In fact, since $f(x, 0)=0$ it follows that 0 is a solution of $\left(P_{\lambda, \mu}\right)$ for every $\lambda, \mu$. Hence, $\lambda=0$ is a bifurcation point for problem $\left(P_{\lambda, \mu}\right)$, in the sense that $(0,0)$ belongs to the closure in $W_{0}^{1, p}(\Omega) \times \mathbb{R}$ of the set

$\left\{(u, \lambda) \in W_{0}^{1, p}(\Omega) \times\right] 0,+\infty\left[: u\right.$ is a weak solution of $\left.\left(P_{\lambda, \mu}\right), u \neq 0\right\}$.

Anyway, also when $f(x, 0) \neq 0$ and $f$, in addition to assumption $(3)$, satisfies the growth condition

$$
|f(x, \xi)| \leq a\left(1+|\xi|^{q}\right)
$$

for every $\xi \in \mathbb{R}$ and a.e. in $\Omega$, where $a>0$ and $q<\frac{n(p-1)+p}{n-p}$, the statements of Theorem 3.1 are still true.

Here is an example of application of Theorem 3.1.

EXAMPLE 1 . Let $\Omega$ be a bounded open subset of $\mathbb{R}^{n}$ with $n \geq 2,1<$ $p<n$ and $\alpha, \beta: \Omega \rightarrow \mathbb{R}$ two continuous and bounded functions. Assume that $\sup _{\Omega} \alpha>0$ and that $\beta$ is a positive function with $\inf _{\Omega} \beta>0$. Then for each $\mu \in\left[0, H\left[\right.\right.$ there exists a positive number $\lambda_{\mu}^{*}$ such that the problem

$$
\left(\widetilde{P}_{\lambda, \mu}\right) \quad\left\{\begin{array}{l}
-\Delta_{p} u=\frac{\mu}{|x|^{p}}|u|^{p-2} u+\lambda\left[\alpha(x)|u|^{r-2} u+\beta(x)|u|^{s-2} u\right] \quad \text { a.e. in } \Omega \\
u_{\mid \partial \Omega}=0
\end{array}\right.
$$

with $1<r<p$ and $p<s<p^{*}$ admits a nonzero weak solution $u_{\lambda}$ in $W_{0}^{1, p}(\Omega)$. Moreover, $\lim _{\lambda \rightarrow 0^{+}}\left\|u_{\lambda}\right\|=0$ and the energy functional related to problem $\left(\widetilde{P}_{\lambda, \mu}\right)$ is negative and decreasing in $] 0, \lambda_{\mu}^{*}[$.

To prove this, we can apply Theorem 3.1 with

$$
f(x, \xi)=\alpha(x)|\xi|^{r-2} \xi+\beta(x)|\xi|^{s-2} \xi
$$

for every $(x, \xi) \in \bar{\Omega} \times \mathbb{R}$.

It is easy to verify that condition (1) holds. Denote by $B_{\varrho}$ the open ball centred at $x_{0}$ with radius $\varrho$, and let $\varrho$ be such that $B_{\varrho} \subseteq \Omega$ and $\min _{B_{\varrho}} \alpha>0$. If we put $D=B=B_{\varrho}$, a simple computation shows that

$$
\lim _{\xi \rightarrow 0^{+}} \frac{\inf _{x \in B} \int_{0}^{\xi} f(x, t) d t}{|\xi|^{p}} \geq \frac{\min _{B} \alpha}{r} \lim _{\xi \rightarrow 0^{+}} \frac{1}{|\xi|^{p-r}}=+\infty .
$$

Hence all the assumptions of Theorem 3.1 are verified and the conclusion follows.

REMARK 2. We point out that the energy functional $J_{\lambda, \mu}$ related to problem $\left(\widetilde{P}_{\lambda, \mu}\right)$ is not coercive. In particular, it is unbounded from below. 
In fact, if we fix $v \in W_{0}^{1, p}(\Omega)$ and $\tau \in \mathbb{R}$, then

$$
\begin{aligned}
J_{\lambda, \mu}(\tau v)= & \frac{\tau^{p}}{p}\|v\|^{p}-\frac{\mu \tau^{p}}{p}\|v\|^{p} \\
& -\lambda\left[\frac{\tau^{r}}{r}\left(\int_{\Omega} \alpha(x) d x\right)\|v\|_{r}^{r}+\frac{\tau^{s}}{s}\left(\int_{\Omega} \beta(x) d x\right)\|v\|_{s}^{s}\right] .
\end{aligned}
$$

So, as $s>p>r$, it follows that $\lim _{\tau \rightarrow+\infty} J_{\lambda, \mu}(\tau v)=-\infty$.

Let now $\Omega$ be a bounded open subset of $\mathbb{R}^{n}$ and consider, for each $\lambda, \mu \in$ $\mathbb{R}$, the functional $I_{\lambda, \mu}: X \rightarrow \mathbb{R}$ defined by putting

$$
I_{\lambda, \mu}(u)=\frac{1}{p} \int_{\Omega}|\nabla u(x)|^{p} d x-\frac{\mu}{p}\left(\int_{\Omega}|u(x)|^{p^{*}} d x\right)^{p / p^{*}}+\lambda \Phi(u)
$$

for each $u \in X$.

Taking into account the Sobolev inequality

$$
\left(\int_{\Omega}|u(x)|^{p^{*}} d x\right)^{1 / p^{*}} \leq \frac{1}{S^{1 / p}}\left(\int_{\Omega}|\nabla u(x)|^{p} d x\right)^{1 / p},
$$

where $u \in X$ and $S$ is the best constant in the Sobolev inclusion (see [7]), and observing that $I_{\lambda, \mu}$ is the energy functional related to problem $\left(P_{\lambda, \mu}^{*}\right)$ introduced in the introduction, in analogy to Theorem 3.1, we can state the following

TheOREm 3.2. Let $f: \Omega \times \mathbb{R} \rightarrow \mathbb{R}$ be a Carathéodory function satisfying the assumptions of Theorem 3.1. Then for every $\mu \in[0, S[$ there exists a positive number $\nu_{\mu}^{*}$ such that for every $\left.\lambda \in\right] 0, \nu_{\mu}^{*}\left[\right.$ problem $\left(P_{\lambda, \mu}^{*}\right)$ admits a nonzero weak solution $u_{\lambda}$ in $W_{0}^{1, p}(\Omega)$. Moreover,

$$
\lim _{\lambda \rightarrow 0^{+}}\left\|u_{\lambda}\right\|=0
$$

and the function $\lambda \mapsto I_{\lambda, \mu}\left(u_{\lambda}\right)$ is negative and decreasing in $] 0, \nu_{\mu}^{*}[$.

\section{References}

[1] D. Arcoya and L. Boccardo, Some remarks on critical point theory for nondifferentiable functionals, NoDEA Nonlinear Differential Equations Appl. 6 (1999), 79-100.

[2] I. Ekeland and N. Ghoussoub, Selected new aspects of the calculus of variations in the large, Bull. Amer. Math. Soc. 39 (2002), 207-265.

[3] J. P. García Azorero and I. Peral, Hardy inequalities and some critical elliptic and parabolic problems, J. Differential Equations 144 (1998), 441-476.

[4] N. Ghoussoub and C. Yuan, Multiple solutions for quasi-linear PDEs involving the critical Sobolev and Hardy exponents, Trans. Amer. Math. Soc. 352 (2000), 57035743.

[5] E. Montefusco, Lower semicontinuity of functionals via the concentration-compactness principle, J. Math. Anal. Appl. 263 (2001), 264-276. 
[6] B. Ricceri, A general variational principle and some of its applications, J. Comput. Appl. Math. 113 (2000), 401-410.

[7] G. Talenti, Best constant in Sobolev inequality, Ann. Mat. Pura Appl. 110 (1976), 353-372.

Dipartimento di Matematica

e Informatica

Università di Catania

Viale A. Doria 6

95125 Catania, Italy

E-mail: ffaraci@dmi.unict.it
Dipartimento di Matematica

Università di Messina Salita Sperone 31 98166 Sant'Agata, Messina, Italy E-mail: livrea@dipmat.unime.it

Reçu par la Rédaction le 9.1.2003 